\title{
RNA: master or servant?
}

\section{KARLA M. NEUGEBAUER}

Yale University, New Haven, Connecticut 06520, USA
RNA has sequence and structure that give it great versatility. It can expose side chains in single stranded regions for protein interaction or translation. It can hide in complicated stem loops. It can be catalytic. These are some of the properties that cause us to love RNA as a molecule. However well this is appreciated by the wider community of biologists, RNA is still viewed as a messenger racing barefoot between the realms of two great kings. Perhaps we have this unconscious bias about RNA, because these are the fairytales we grew up with. Is it possible that RNA has even greater capacity to organize cells than we have imagined already?

Look at RNA in cells. Ramon y Cajal did this in the 19th century when he stained sections of the vertebrate nervous system with silver. Silver deposits on RNA-rich structures revealed two nuclear structures: nucleoli and accessory bodies (now called Cajal bodies). Today we know that nuclear and cytoplasmic bodies-including nucleoli, Cajal bodies, histone locus bodies, nuclear speckles, paraspeckles, P bodies, and $\mathrm{P}$ granules-are packed with different kinds of RNA: long non-coding RNAs, spliceosomal snRNAs, snoRNAs and mRNAs. RNA-rich bodies play important regulatory roles in RNA biogenesis and stability, by making processes like snRNP assembly more efficient. Proteins have been identified that specifically target RNA to one or another body, suggesting that RNA is the passenger in a cart drawn by a team of heaving proteins.

On the contrary, RNA drives the formation of many of these bodies. Nucleoli form at the sites of rDNA transcription, Cajal bodies at active snRNA genes, histone locus bodies at histone gene clusters, and paraspeckles at active sites of NEAT1 transcription. In these cases, nascent RNA emerging from the DNA template can be seen as the Velcro that attracts other sticky molecules, such as RNA-binding proteins and other RNA-containing complexes like snoRNPs that can build on to nascent RNA through base-pairing interactions. Similarly, snRNPs can base-pair with nascent pre-mRNA, accounting for co-transcriptional assembly of the spliceosome. Co-transcriptional RNA processing — capping, splicing, editing, and polyadenylation-means that these machineries are attracted to nascent RNA and concentrate at each gene.

Corresponding author: karla.neugebauer@yale.edu

Article and publication date are at http://www.rnajournal.org/cgi/doi/ 10.1261/rna.051250.115. Freely available online through the RNA Open Access option.
Because of the specificity of these interactions, genes differ in the constellation of associated factors when transcription is active. Each gene, therefore, might be viewed as a mini-nuclear body.

What about mRNA? The current view is that export receptors take mRNA out of the nucleus and, once in the cytoplasm, other proteins may localize mRNA to particular subcellular regions. High throughput in situ hybridization studies, like those performed by Lecuyer and Krause, have revealed that the majority of mRNAs are localized within eukaryotic cells. Why? mRNA localization is essential for the maintenance of cellular asymmetry. Molecular motors can transport mRNAs on actin filaments and/or microtubules to concentrate mRNA in presynaptic terminals of neurons or into the daughter cell of yeast at cell division. Actin mRNA localizes to the leading edge of fibroblasts. Pioneering work from the Singer lab focused on and identified a cis-acting "zip code" responsible for binding specific proteins and guiding RNA to the correct site. Since then, many zip codes have been identified, even in E. coli where mRNA localization correlates with the region of highest translation. These findings focus our attention on the notion that mRNA localization serves protein function.

Consider the two sides to the double stranded RNA binding protein Staufen. Staufen is required for oskar localization to the posterior pole of the fruitfly oocyte and later for bicoid localization to the anterior pole of the embryo. From this observation, one may hypothesize that Staufen's ability to localize RNA depends on developmental stage-specific regulation. On the other hand, St Johnston's lab showed that Staufen localization to either pole depends on bicoid and oskar mRNAs! Therefore, rather than thinking about what proteins can do for RNA, let's think about what RNAs can do for protein.

If the majority of mRNAs localize to different zones of cytoplasm, perhaps proteins and other molecules that associate with these RNAs are also differentially localized in cytoplasm. We may be blind to many cellular compartments created by RNA for any number of reasons, the most obvious being that we haven't looked yet. The important feature of localization is not necessarily just about cellular position. Long and highly structured RNAs, like the bicoid 3' UTR, could easily serve

(C) 2015 Neugebauer This article, published in RNA, is available under a Creative Commons License (Attribution-NonCommercial 4.0 International), as described at http://creativecommons.org/licenses/by-nc/4.0/. 
as a platform for bringing other molecules together. For example, the Hentze and Landthaler groups have recently shown that metabolic enzymes bind RNA. Perhaps clustering these enzymes on an RNA platform modifies their activities or efficacy, as we have seen before with nuclear bodies. I wonder if there may be more cytoplasmic bodies than we know about? Smaller RNA-mediated aggregates that look like point sources of light? Gradients of RNA-dependent activity across cytoplasm?

One of my very favorite experiments in biology was published in the RNA journal. In it, the Yarus lab evolved an RNA that could bind the amino acid tryptophan and a distinct RNA that could bind phospholipid bilayers. When these two RNAs were fused together, a membrane transporter capable of turning over tens of thousands of amino acids per RNA was created (Janas, Janas and Yarus, 2004, RNA 10:1541). Although there are many experiments that display the remarkable activities of RNA, this paper convinced me that RNA can do absolutely anything.

We have not seen the end of what RNA can do in cells. One reason we lack information about the roles of RNA in cellular regulation is that we have known about proteins for a longer period of time. Protein activities and localization patterns captured our imagination first, and we referred to proteins as markers for this or that compartment before we realized that RNAs are also markers and could equally well drive function. We now have examples of RNAs that dominate cellular regulation. The essential roles long non-coding RNAs, such as XIST in X-inactivation and NEAT1 in paraspeckle formation, are examples of the power of RNA in vivo. We are still in a phase of cataloging the RNAs expressed in cells and developing assays to determine their functions. In five or ten years, examples of RNA-mediated cellular organization may seem less like exceptions and more like rules. 

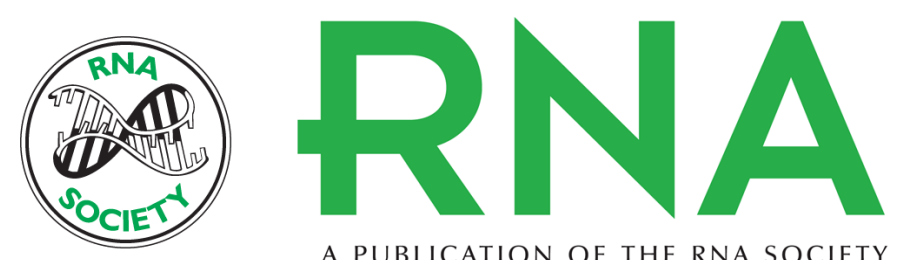

A PUBLICATION OF THE RNA SOCIETY

\section{RNA: master or servant?}

Karla M. Neugebauer

RNA 2015 21: 701-702

Open Access Freely available online through the RNA Open Access option.

Creative This article, published in $R N A$, is available under a Creative Commons License

Commons (Attribution-NonCommercial 4.0 International), as described at

License http://creativecommons.org/licenses/by-nc/4.0/.

Email Alerting Receive free email alerts when new articles cite this article - sign up in the box at the Service top right corner of the article or click here. 